\title{
IN VIVO MAGNETOMYOGRAMS OF SKELETAL MUSCLE
}

\author{
W.L.C. Rutten, A. Wildeman, B.K. van Veen and W. Wallinga \\ Biomedical Engineering Division, Department of electrical Engineering, \\ Twente University, 7500 AE Enschede, The Netherlands.
}

\begin{abstract}
Magnetomyography (MMG) is a new non-invasive technique (inspired by the magnetoneurographic method of Wikswo [1]) to detect action currents in a muscle, which is immersed in a well conducting fluid. The detection coil is of a toroidal shape, with the muscle lead through the center of the coil. For a long muscle which fits tightly in the toroid, it is to be expected that magnetic fields correspond almost completely to the intracellular longitudinal (axial) currents in active muscle fibers. We developed an experimental set-up with specific coils for rat and mouse skeletal muscles which is sensitive enough to detect currents from single motor units.

The technique enables to record stimulated twitch activity in alive muscle as a function of force level, coil position along the muscle, temperature etc.

By simulation with a finite element forward model we calculated action currents under various experimental conditions.
\end{abstract}

\section{MATERIALS AND METHODS}

\section{Sensor system}

Toroidal coils were constructed, using modified Sifferit $(R)$ ring cores with diameters up to $8 \mathrm{~mm}$, a thickness of $0.5 \mathrm{~mm}$ and up to 600 turns. A low input impedance amplifier system with a ten-fold parallel input stage was matched to these coils as optimal as possible. Equivalent input current noise (i.e. the current to be detected in a wire lead through the toroid) typically decreased as a function of increasing frequency (due to the inductive properties of the sensor) and then rose at high frequencies (because of amplifier noise and other effects). With more turns the performance at low frequencies improved, but degraded at high frequencies. The cross over frequency was optimized in a range between about $100 \mathrm{~Hz}$ and 10 $\mathrm{kHz}$.

The minimal input equivalent current noise of the coils is about $10^{-9} \mathrm{~A} / \sqrt{\mathrm{Hz}}$. They have a current noise limit of $10^{-7}$ $10^{-8} \mathrm{~A}$ in a $10 \mathrm{kHz}$ bandwidth, after 100 times averaging. This figure must be compared to the axial currents in muscle fibers and motor units. For example in the rat's Extensor Digitorum Longus (EDL) muscle, calculation shows that one muscle fiber contributes about $2.5 \times 10^{-7}$ Ampere. Consequently, a typical motor unit with 100 fibers contributes $2.5 \times 10^{-5}$ Ampere, which is well above the detection limit (see also discussion)

\section{Set-up and animal preparation}

EDL and tibial muscles in the hindlegs of anaesthetised rats and mouses were exposed over about half their length, so that the coil could be moved freely between tendon and centre of the muscle. The muscle was in an in-vivo condition with normal blood supply and normal neural connections. The free origo tendon was connected to a force transducer and the knee was fixed. Muscle twitches were elicited with a neural hook electrode pair stimulating the peroneal nerve. At the lowest contraction level (one or a few active motor units) about 100 averages were needed, with an interstimulus interval of about one second. Hindleg, muscle and coil were immersed in $80 \mathrm{~cm}^{3}$ Ringer solution.

\section{RESULTS}

By lowering the fluid level it was verified that the bath outside the muscle was large enough to serve as a good conductor, which is an essential prerequisite of this method. It appeared that responses started to diminish only until the fluid level approached the top of the coil within about a millimeter.

Figure 1 shows two examples of responses in the rat EDL muscle at intermediate and low twitch force levels (see fig. 2 for the actual force level). These and other have a clear biphasic shape and a width, which depends on force level and species.

Figure 2 summarizes current (peak-to-peak values of the biphasic shape) versus force relationships in rat tibial and EDL muscle. The results show an almost linear current to force relationship, from the single motor unit force level $(0.03 \mathrm{~N})$ to the maximum force level $(0.5-1 \mathrm{~N})$. Figure 3 is the finite element simulated single fibre MMG of the EDL muscle. Parameters are the same as in [2], except the conduction velocity $\mathrm{v}=5 \mathrm{~m} / \mathrm{s}$. Cancellation of currents due to return currents in the muscle inside the coil appeared to give a reduction of $10 \%$.

\section{DISCUSSION}

The biphasic shape of the responses (figures 1 and 2) is a strong indication that the signal results from the axial intracellular current $i_{z}$. In a core conductor model one has

$$
\mathrm{i}_{\mathbf{z}}=\frac{\sigma_{\mathrm{i}} \mathrm{A}}{\mathrm{v}} \frac{\partial \mathrm{v}_{\mathrm{i}}}{\partial \mathrm{t}}
$$




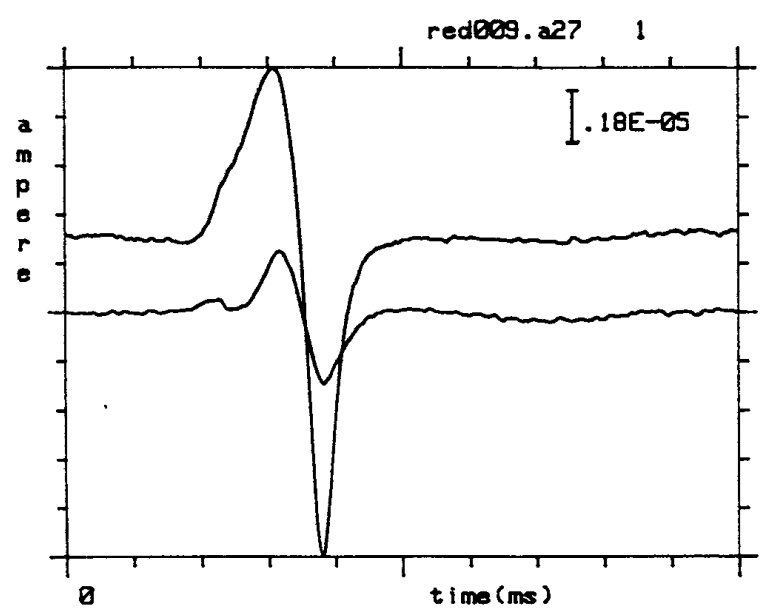

Figure 1. Biphasic magnetomyograms of rat EDL muscle.

Two responses, at two different twitch force levels (see also fig. 2), are shown. The sensor is a toroild coil around the muscle, midway between origo tendon and muscle belly. The vertical scale is the current in the muscle which yields the circular component of the magnetic field, picked up by the toroid.

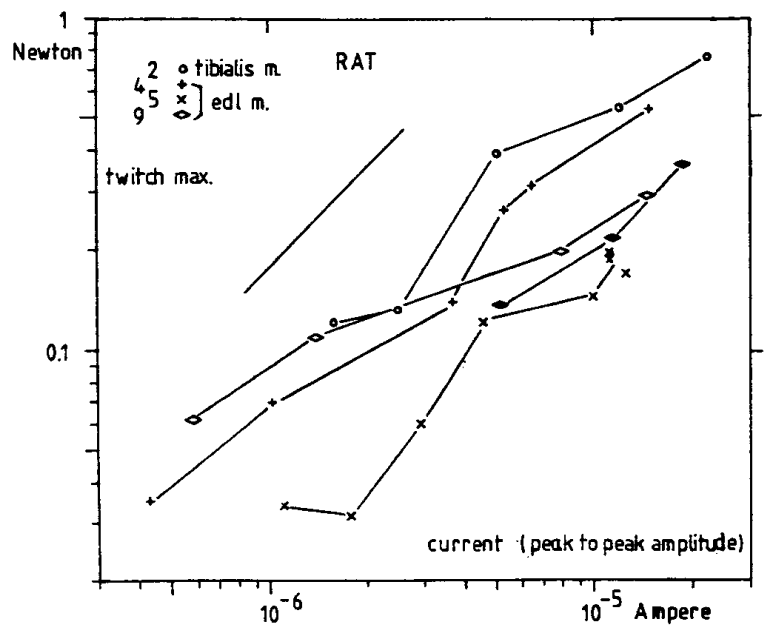

Figure 2. Peak-to peak currents of magnetomyograms, such as in figures 1 and 2, versus corrresponding twitch response amplitudes for rat $E D L$ and tibial muscles. in which $\sigma_{i}$ is the intracellular conductivity, $\mathrm{A}$ is the fiber cross section and $v$ is the conduction velocity. $V_{i}$ is the intracellular action potential (ap). Taking $v_{i}$ as an analytical approximation [2]

$$
\mathrm{v}_{\mathrm{i}}=\alpha \mathrm{t}^{3} \mathrm{e}^{-\beta \mathrm{t}}-\gamma
$$

one calculates for one EDL muscle fibre a biphasic shape with a peak to peak current of $2.5 \times 10^{-7}$ Ampere. This is in good agreement with the simulated result (fig. 3 ). So, with about 100 fibres in one EDL motor unit one would expect about

$2.5 \times 10^{-5} \mathrm{~A}$ for the mu response. This contrasts with the observed value of $4 \times 10^{-7} \mathrm{~A}$ (fig. 2). The difference may be due to several causes such as 1) cancellation of single fibre currents due to different timing on sets in one motor unit.

2) reduction effects due to finite dimensions of muscle, bath and coil. 3) position of the mu inside the muscle cross section. Future simulations must show the precise amount of cancellation due to volume currents.

\section{REFERENCES}

[1] J.P. Wikswo jr., P.C. Samson and R.P. Giffard. A low-noise, low input impedance amplifier for magnetic measurements of nerve action currents. IEEE Trans. Biomed Eng. BME-30, pp. 215-221: 1983.

[2] B.A. Albers, W.L.C. Rutten, W. Wallinga and H.B.K. Boom. Microscopic and macroscopic volume conduction in skeletal muscle tissue, applied to simulation of single-fibre action potentials.

Medical and Biological Engineering and Computing, pp. 605-616: 1988 .

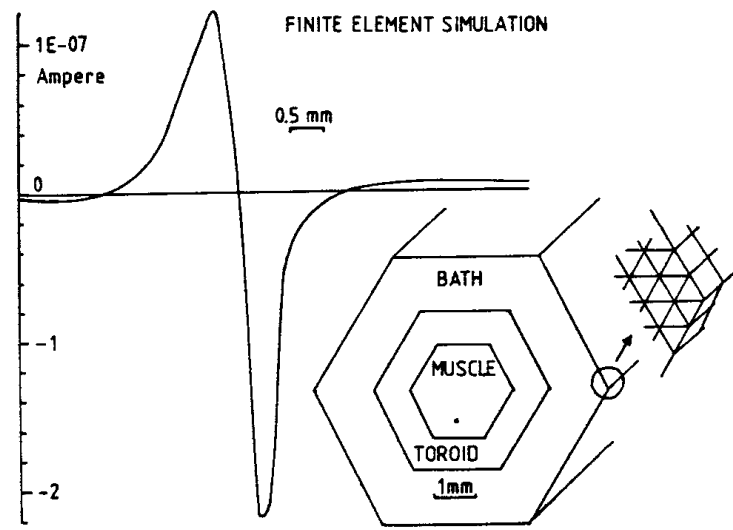

Figure 3. Simulated $M M G$ response in a finite element model. The active equivalent single fibre is situated excentrically in the muscle. The two inserts show the model structure (sectioned at the toroid position, midway origo tendon and muscle belly) and the element shape. 